\title{
Long-term Postoperative Nutritional Status Affects Prognosis Even After Infectious Complications in Gastric Cancer
}

\author{
JUN KIUCHI ${ }^{1 *}$, SHUHEI KOMATSU ${ }^{1 *}$, TOSHIYUKI KOSUGA ${ }^{1}$, TAKESHI KUBOTA ${ }^{1}$, \\ KAZUMA OKAMOTO ${ }^{1}$, HIROTAKA KONISHI $^{1}$, ATSUSHI SHIOZAKI ${ }^{1}$, \\ HITOSHI FUJIWARA ${ }^{1}$, DAISUKE ICHIKAWA ${ }^{2}$ and EIGO OTSUJI ${ }^{1}$ \\ ${ }^{1}$ Division of Digestive Surgery, Department of Surgery, Kyoto Prefectural University of Medicine, Kyoto, Japan; \\ ${ }^{2}$ Department of Digestive Surgery, Yamanashi University, Chuo, Japan
}

\begin{abstract}
Aim: This study was designed to investigate the clinical impact of postoperative serum albumin level on severe postoperative complications (SPCs) and prognosis. Materials and Methods: Data for a total of 728 consecutive patients who underwent curative gastrectomy for gastric cancer between 2004 and 2013 were retrospectively analyzed. From these patients, a propensity score-matched analysis was performed based on 14 clinicopathological and surgical factors. Results: Short-term decrease in postoperative serum albumin level was not associated with the occurrence of SPCS. Regarding long-term decrease in serum albumin level, a decrease of $\geq 0.5 \mathrm{~g} / \mathrm{dl}$ at 3 months did not affect the long-term survival of patients without SPCs, but was related to a significantly poorer prognosis in patients with SPCs. By multivariate analysis, long-term decrease of serum albumin level was an independent prognostic factor in patients with SPCs. Conclusion: Longterm postoperative nutritional status as shown by a low level of albumin was related to prognosis in patients with SPCs.
\end{abstract}

Gastric cancer is one of the most common causes of death from cancer worldwide (1). Recent advances in diagnostic techniques have contributed to the earlier detection of gastric cancer, and less invasive endoscopic or surgical techniques, standardized surgical procedures, perioperative management techniques, and effective novel agents for chemotherapy

\footnotetext{
*These Authors contributed equally to this study.

Correspondence to: Shuhei Komatsu MD, Ph.D., Division of Digestive Surgery, Department of Surgery, Kyoto Prefectural University of Medicine, 465 Kajii-cho, Kawaramachihirokoji, Kamigyo-ku, Kyoto 602-8566, Japan. Tel: +81 752515527, Fax: +81 752515522, e-mail: skomatsu@koto.kpu-m.ac.jp
}

Key Words: Serum albumin decrease, albumin loss, prognosis, nutritional status, gastric cancer, postoperative complications. have drastically reduced the morbidity and mortality rates of patients with gastric cancer (2-4). However, postoperative complications and tumor recurrence after gastrectomy occur in some patients even today; therefore, further improvement of treatment in patients with gastric cancer is required.

A lower preoperative nutritional status has been reported to be associated with the occurrence of postoperative complications and a long-term poorer prognosis (5-7). The preoperative serum albumin level reflects the nutritional status and is used as a pivotal factor in the Glasgow Prognostic Score, which can predict the prognosis of patients with cancer (8-10). However, to our knowledge, there have been no reports about whether the postoperative serum albumin level influences the occurrence of postoperative complications and long-term outcomes.

In this study, the association between a decrease in perioperative serum albumin level and the occurrence of postoperative complications was evaluated and the long-term prognostic impact of postoperative serum albumin level at 3 months after surgery may investigated in patients with gastric cancer. Our results provide further evidence that the postoperative serum albumin level might be an indicator for prognosis and that nutritional maintenance may contribute to improving prognosis in patients with gastric cancer.

\section{Materials and Methods}

Patients and operative procedures. This study was institutionally and comprehensively in accordance with ethical standards of the Kyoto Prefectural University of Medicine and with the Helsinki Declaration. The patients provided their informed written consent prior to study enrolment. We retrospectively analyzed data for 728 consecutive patients who underwent curative gastrectomy with lymphadenectomy for gastric cancer at the Division of Digestive Surgery, Department of Surgery, Kyoto Prefectural University of Medicine between 2004 and 2013. Patients with stage IV cancer were excluded from this study. Based on the preoperative diagnosis using gastric endoscopy and computed tomographic scans, total or distal gastrectomy with sufficient lymphadenectomy was performed, mainly according to the Japanese guidelines for the treatment of gastric cancer (11). Patients 
with clinical T1 and N0 tumors underwent D1 or D1 with lymphadenectomy, and patients with clinical T2 or more advanced tumors and those with $\mathrm{N} 1$ or more advanced tumors underwent either D2 or D2 with lymphadenectomy. In the D2 dissection, the perigastric lymph nodes and all second-tier lymph nodes were completely retrieved. Depending on the location of the tumor, additional lymphadenectomy was performed along the distal side of the splenic artery (no.11d) and at the splenic hilum (no.10), together with splenectomy or splenectomy with distal pancreatectomy $(11,12)$.

Three major infectious complications were defined as severe postoperative complications (SPCs): anastomotic leakage, pancreatic fistula, and intra-abdominal abscess, all of which are classified as being more than grade II in the Clavien-Dindo classification (13). In the present study, the impact of the serum albumin decrease following gastrectomy for gastric cancer on the occurrence of SPCs and prognosis was evaluated. Considering the selection bias from potential confounders, a propensity score-matched analysis was carried out to compare the outcomes between patients with and without SPCs. The variables for calculating the propensity score were sex, age at surgery, presence of emaciation or obesity, presence of anemia, preoperative serum albumin level, extent of lymphadenectomy, surgical approach, surgical form, pathological $\mathrm{T}$ factor, pathological $\mathrm{N}$ factor, pathological tumor stage by TNM classification (12), presence of concurrent diabetes mellitus, and presence of concurrent hepatic disease.

In this study, firstly the association between serum albumin decrease and the occurrence of severe postoperative infectious complications was investigated. Next, the decrease in serum albumin level and the prognostic impact of a prolonged low serum albumin level was compared between patients with and without SPCs. In this study, a decrease of $0.5 \mathrm{~g} / \mathrm{dl}$ or greater at postoperative day 90 was used as a cut-off for a prolonged low serum albumin level.

Statistical analysis. Statistical analysis was performed using JMP version 11 (ASA Institute, Cary, NC, USA). The chi-square test and Fisher's exact probability test were performed for categorical variables, and the Student's $t$-test and the Mann-Whitney $U$-test for unpaired data of continuous variables were performed to compare the clinicopathological characteristics between the two groups. Overall and disease-free survival curves, which were measured from surgery, were estimated using the Kaplan-Meier method, and statistical differences were examined using the log-rank test. Univariate and multivariate survival analyses were performed using the likelihood ratio test of the stratified Cox proportional hazards model. Values of $p<0.05$ derived from two-tailed test were considered statistically significant.

\section{Results}

Comparison of clinicopathological characteristics between patients with and without SPCs after propensity score matching. A total of 45 out of 728 patients $(6.2 \%)$ had SPCs after gastrectomy for gastric cancer. Eighty-six patients, consisting of 43 patients with postoperative infectious complications and 43 patients without were included in the analysis after propensity score matching. Table I shows the clinicopathological characteristics of these two groups; there were no significant differences in patient background between the two groups.
Table I. Comparison of clinicopathological characteristics between patients with and without severe postoperative complications (SPC) after propensity score matching.

\begin{tabular}{|c|c|c|c|}
\hline \multirow[b]{2}{*}{ Variable } & \multicolumn{2}{|c|}{$\mathrm{SPC}$} & \multirow[b]{2}{*}{$p$-Value ${ }^{\mathrm{a}}$} \\
\hline & $\begin{array}{l}\text { With } \\
(n=43)\end{array}$ & $\begin{array}{l}\text { Without } \\
(n=43)\end{array}$ & \\
\hline \multicolumn{4}{|l|}{ Gender } \\
\hline Female & $9(47 \%)$ & $10(53 \%)$ & 1.000 \\
\hline Male & $34(51 \%)$ & $33(49 \%)$ & \\
\hline \multicolumn{4}{|l|}{ Age } \\
\hline$<65$ Years & $12(43 \%)$ & $16(57 \%)$ & 0.490 \\
\hline$\geq 65$ Years & $31(53 \%)$ & $27(47 \%)$ & \\
\hline \multicolumn{4}{|l|}{ Emaciation, BMI } \\
\hline$\geq 18.5 \mathrm{~kg} / \mathrm{m}^{2}$ & $40(51 \%)$ & $39(49 \%)$ & 1.000 \\
\hline$<18.5 \mathrm{~kg} / \mathrm{m}^{2}$ & $3(43 \%)$ & $4(57 \%)$ & \\
\hline \multicolumn{4}{|l|}{ Obesity, BMI } \\
\hline$<25 \mathrm{~kg} / \mathrm{m}^{2}$ & $31(48 \%)$ & $33(52 \%)$ & 0.805 \\
\hline$\geq 25 \mathrm{~kg} / \mathrm{m}^{2}$ & $12(55 \%)$ & $10(45 \%)$ & \\
\hline \multicolumn{4}{|l|}{ Anemia, $\mathrm{Hb}$} \\
\hline$\geq 8 \mathrm{~g} / \mathrm{dl}$ & $42(50 \%)$ & $42(50 \%)$ & 1.000 \\
\hline$<8 \mathrm{~g} / \mathrm{dl}$ & $1(50 \%)$ & $1(50 \%)$ & \\
\hline \multicolumn{4}{|l|}{ Preoperative albumin } \\
\hline$\geq 3.0 \mathrm{~g} / \mathrm{dl}$ & $42(50 \%)$ & $42(50 \%)$ & 1.000 \\
\hline$<3.0 \mathrm{~g} / \mathrm{dl}$ & $1(50 \%)$ & $1(50 \%)$ & \\
\hline \multicolumn{4}{|l|}{ Lymphadenectomy } \\
\hline$<\mathrm{D} 2$ & $22(46 \%)$ & $26(54 \%)$ & 0.515 \\
\hline$\geq \mathrm{D} 2$ & $21(55 \%)$ & $17(45 \%)$ & \\
\hline \multicolumn{4}{|l|}{ Surgical approach } \\
\hline Laparotomy & $6(50 \%)$ & $6(50 \%)$ & 1.000 \\
\hline Laparoscopic & $37(50 \%)$ & $37(50 \%)$ & \\
\hline \multicolumn{4}{|l|}{ Surgical method } \\
\hline Partial gastrectomy & $25(46 \%)$ & $29(54 \%)$ & 0.503 \\
\hline Total gastrectomy & $18(56 \%)$ & $14(44 \%)$ & \\
\hline \multicolumn{4}{|l|}{ T Factor } \\
\hline $\mathrm{T} 1, \mathrm{~T} 2$ & $32(51 \%)$ & $31(49 \%)$ & 1.000 \\
\hline $\mathrm{T} 3, \mathrm{~T} 4$ & $11(48 \%)$ & $12(52 \%)$ & \\
\hline \multicolumn{4}{|l|}{ N Factor } \\
\hline N0, N1 & $32(48 \%)$ & $34(52 \%)$ & 0.799 \\
\hline $\mathrm{N} 2, \mathrm{~N} 3$ & $11(55 \%)$ & $9(45 \%)$ & \\
\hline \multicolumn{4}{|l|}{ TNM stage } \\
\hline I & $22(50 \%)$ & $22(50 \%)$ & 1.000 \\
\hline II or III & $21(50 \%)$ & $21(50 \%)$ & \\
\hline \multicolumn{4}{|l|}{ Diabetes mellitus } \\
\hline No & $36(51 \%)$ & $34(49 \%)$ & 0.782 \\
\hline Yes & $7(44 \%)$ & $9(56 \%)$ & \\
\hline \multicolumn{4}{|l|}{ Hepatic disease } \\
\hline No & $38(50 \%)$ & $38(50 \%)$ & 1.000 \\
\hline Yes & $5(50 \%)$ & $5(50 \%)$ & \\
\hline
\end{tabular}

aUnivariate analysis was assessed using Chi-square test and Fisher's exact probability test.

Decrease in serum albumin level at postoperative days 1 and 3. The decrease in serum albumin level between patients with and without SPCs at postoperative days 1 and 3 was compared but there was no significant difference between the two groups (Figure 1). 
A

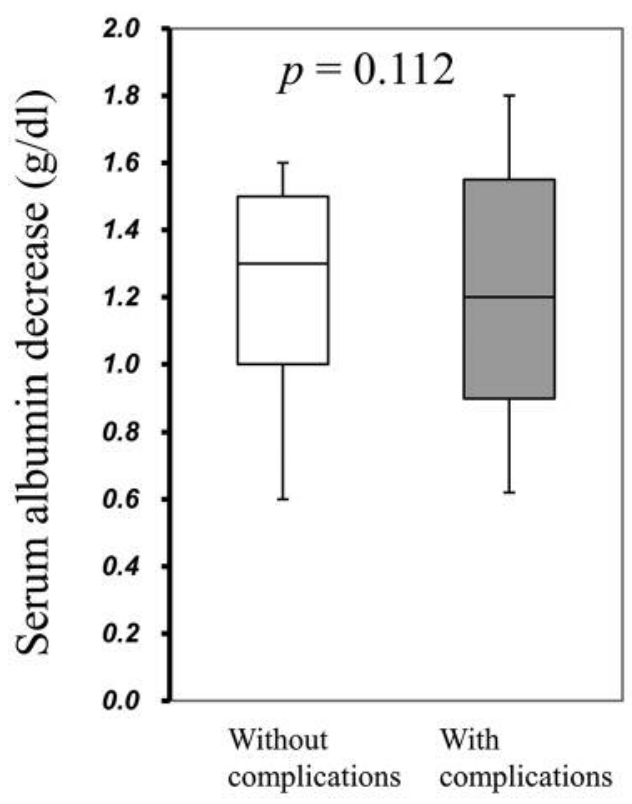

B

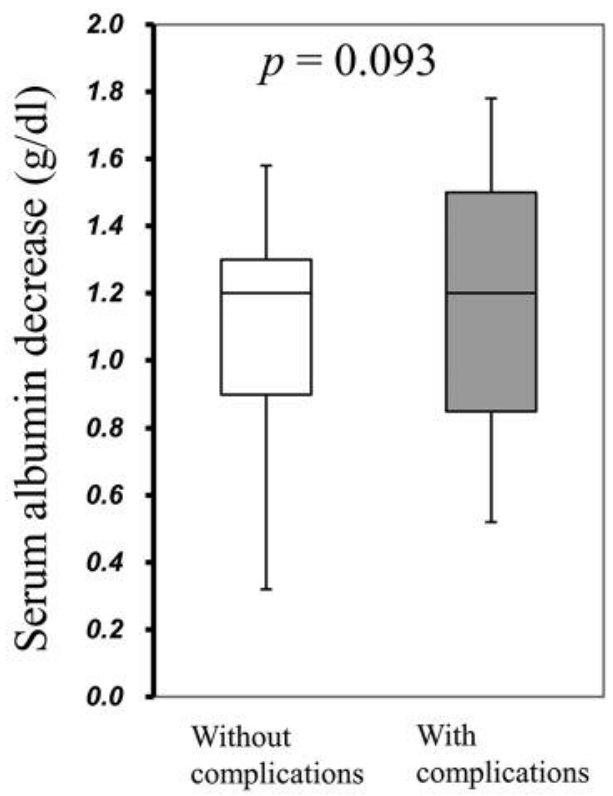

Figure 1. Comparison of the decrease in serum albumin level between patients with and without severe postoperative complications on postoperative days $1(A)$ and $3(B)$. The boxes, lines and bars respectively indicate lower and upper quartile, median values, and minimum and maximum values. The decrease in serum albumin level at both time points did not significantly differ between the two groups.

Prognostic impact of a prolonged serum albumin decrease. Next, we investigated the prognostic impact of a prolonged serum albumin decrease. In patients without SPCs, there was no significant difference in overall (Figure 2A) or diseasefree survival (Figure 2B) between patients with and those without a prolonged serum albumin decrease $(p=0.547$ and $p=0.785$, respectively). However, in patients with SPCs, those with a prolonged low serum albumin level had significantly poorer overall (Figure 3A) and disease-free survival (Figure 3B) ( $p=0.040$ and $p=0.041$, respectively). Univariate and multivariate analyses using Cox's proportional hazards model revealed that a prolonged low serum albumin level was an independent prognostic factor in patients with SPCs (hazard ratio=7.28, 95\% confidence interval=1.132-187.5; $p=0.042$ ) (Table II).

\section{Discussion}

Patients with gastric cancer following gastrectomy often develop malnutrition. In this study, we demonstrated that a prolonged postoperatively low serum albumin level contributed to a significantly poorer prognosis in patients with SPCs. Furthermore, this characteristic was an independent prognostic factor by multivariate analysis in patients with SPCs. These results suggest that postoperative nutritional maintenance may be necessary to improve a poor
Table II. Univariate analysis of overall survival after surgery using the Cox's proportional hazard model in patients with severe postoperative complications. The multivariate analysis using Cox's proportional hazard model confirmed the prognostic value of postoperative serum albumin decrease $(H R=728,95 \% C I=1.132-187.5, p=0.042)$.

\begin{tabular}{lccc}
\hline Variable & Comparison & $\mathrm{n}$ & $p$-Value \\
\hline Gender & Male vs. female & 34 vs. 9 & 0.040 \\
Age & $>65$ vs. $<65$ Years & 31 vs. 12 & 0.796 \\
T-Stage* & T3, 4 vs. T1, 2 & 11 vs. 32 & 0.006 \\
N-Stage & $\mathrm{N} 2,3$ vs. N0,1 & 11 vs. 32 & 0.112 \\
Lymphatic invasion (ly) & 2,3 vs. 0,1 & 6 vs. 37 & 0.789 \\
Venous invasion (v) & 2,3 vs. v0, 1 & 13 vs. 30 & 0.011 \\
Decrease in albumin level* & $\geq 0.5$ vs. $<0.5 \mathrm{~g} / \mathrm{dl}$ & 20 vs. 23 & 0.040 \\
\hline
\end{tabular}

*TNM classification.**At postoperative day 90 compared to

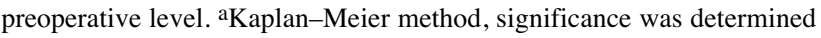
by log-rank test.

prognosis following SPCs in patients with gastric cancer. As far as we are aware, this is the first report that a long-term low postoperative serum albumin level can affect prognosis in patients with gastric cancer.

Serum albumin level has often been used as an indicator of nutritional status. There are many reports that a low preoperative nutritional status might be associated with the 

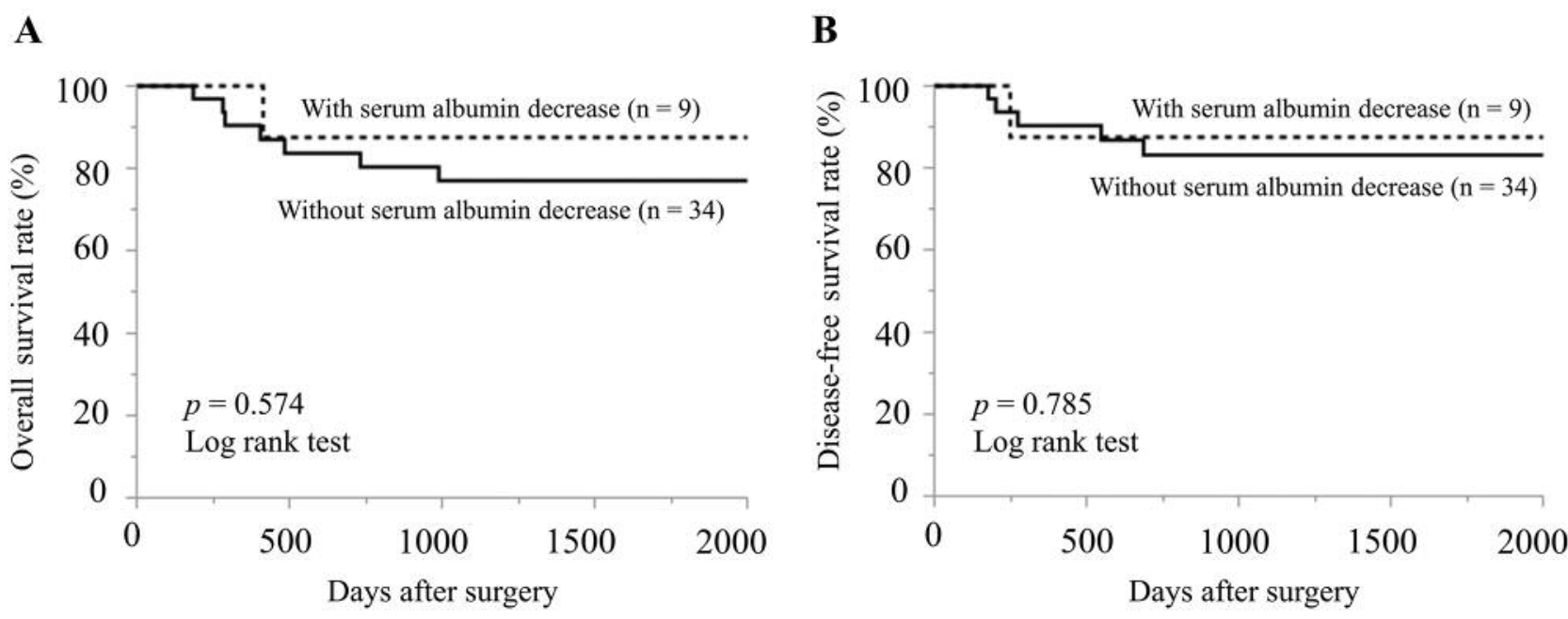

Figure 2. Prognostic impact of a prolonged serum albumin decrease in patients without severe postoperative complications. There were no significant difference in overall $(A)$ and disease-free survival $(B)$ between patients with and without a prolonged serum albumin decrease.

A

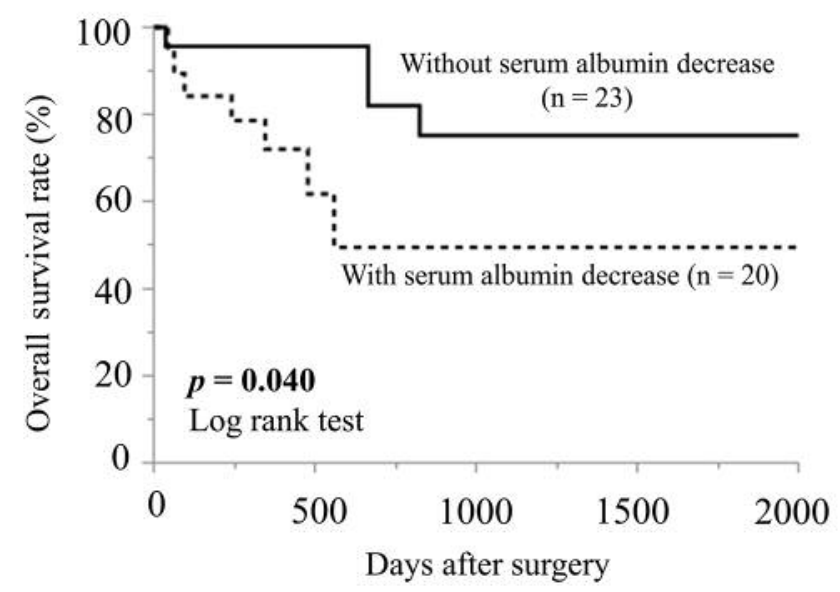

B

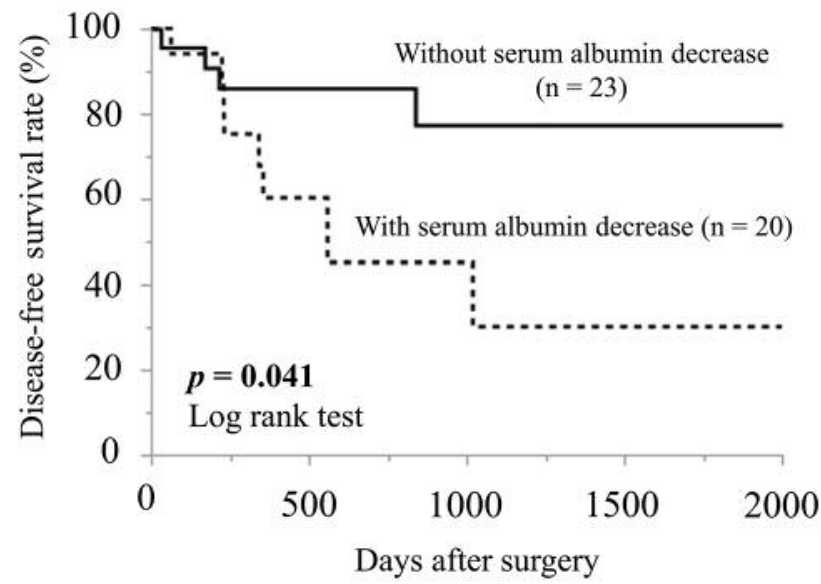

Figure 3. Prognostic impact of a prolonged serum albumin decrease in patients with severe postoperative complications. Both overall (A) and disease-free survival $(B)$ was significantly poorer in patients with a prolonged serum albumin decrease.

occurrence of postoperative complications and a long-term poor prognosis in gastric cancer. (5-7). Migita et al. reported that the preoperative serum albumin level and total lymphocyte count were associated with the occurrence of postoperative complications and long-term outcomes (7). In the Glasgow Prognostic Score, the serum albumin level is used to assess the degree of cachexia and the prognosis of patients with cancer (8-10). These findings prompted us to investigate the clinical impact of postoperative serum albumin level on postoperative complications and prognosis for further application in clinical settings.
We hypothesized that a decrease in serum albumin after gastrectomy might affect the occurrence of postoperative complications and the long-term prognosis. Our findings show that a low perioperative serum albumin level after gastrectomy was not associated with the occurrence of postoperative complications; however, it was related to a poorer prognosis. Regarding the perioperative serum albumin level after gastrectomy, this may be influenced by various factors such as intraoperative bleeding, intra- and postoperative inflammation, and albumin transfusion. Therefore, a short-term decrease in serum albumin level after gastrectomy may not accurately 
reflect the true nutritional status of patients. On the other hand, the long-term serum albumin level is not affected by such surgical factors and may be a reliable indicator of nutritional status.

Several recent studies identified that complications due to postoperative infections can contribute to recurrence and poor outcomes in patients with gastric cancer (14-17). Recent advances in surgical techniques and perioperative management have reduced the occurrence of such complications; however, these complications also occur due to various inevitable reasons. A treatment strategy for avoiding the negative impact of postoperative infectious complications for prognosis is urgently required. Namely, special effort to obtain a better prognosis of patients with postoperative complications might be needed. In this study, we demonstrated that postoperative nutritional status for those with a serum albumin decrease of $<0.5 \mathrm{~g} / \mathrm{dl}$ at 3 months was related to better prognosis even after SPCs in patients with gastric cancer.

Recently, Imamura et al. reported that supplementation of an oral elemental diet reduced postoperative weight loss in patients with gastric cancer undergoing gastrectomy (18). Body weight loss has been reported to be an independent prognostic factor for poorer recurrence-free survival (19) and to be associated with the lower rate of continuation of adjuvant chemotherapy $(19,20)$. Considering the results of this study, supplementation with an oral elemental diet or other nutritional treatment strategy may be necessary not only to reduce postoperative body weight loss but also to improve the poor prognosis of patients with SPCs after gastrectomy. Long-term stable nutrition and treatment may maintain immune competence and suppress recurrence derived from micrometastases after curative surgery $(21,22)$.

One limitation of our study was that the results were retrospectively demonstrated with a small number of patients and variations of the treatment at a single institute. A largescale or multicenter prospective cohort study is warranted to validate the clinical importance of the long-term serum albumin level after gastrectomy.

\section{Conclusion}

Postoperative long-term nutritional status was related to the prognosis in patients with SPCs. Postoperative nutritional maintenance may improve for poor prognosis following complications from postoperative infections in patients with gastric cancer.

\section{References}

1 Ferlay J, Shin HR, Bray F, Forman D, Mathers C and Parkin DM: Estimates of worldwide burden of cancer in 2008: Globocan 2008. Int J Cancer 127(12): 2893-2917, 2010.
2 Kitano S, Shiraishi N, Uyama I, Sugihara K, Tanigawa N and Japanese Laparoscopic Surgery Study Group: A multicenter study on oncologic outcome of laparoscopic gastrectomy for early cancer in japan. Ann Surg 245(1): 68-72, 2007.

3 Komatsu S, Ichikawa D, Kashimoto K, Kubota T, Okamoto K, Konishi H, Shiozaki A, Fujiwara H and Otsuji E: Risk factors to predict severe postoperative pancreatic fistula following gastrectomy for gastric cancer. World J Gastroenterol 19(46): 8696-8702, 2013.

4 Zhang Q, Wang F, Chen ZY, Wang Z, Zhi FC, Liu SD and Bai Y: Comparison of the diagnostic efficacy of white light endoscopy and magnifying endoscopy with narrow band imaging for early gastric cancer: A meta-analysis. Gastric Cancer 19(2): 543-552, 2016.

5 Yamanaka H, Nishi M, Kanemaki T, Hosoda N, Hioki K and Yamamoto M: Preoperative nutritional assessment to predict postoperative complication in gastric cancer patients. JPEN J Parenter Enteral Nutr 13(3): 286-291, 1989.

6 Lien YC, Hsieh CC, Wu YC, Hsu HS, Hsu WH, Wang LS, Huang $\mathrm{MH}$ and Huang BS: Preoperative serum albumin level is a prognostic indicator for adenocarcinoma of the gastric cardia. J Gastrointest Surg 8(8): 1041-1048, 2004.

7 Migita K, Takayama T, Saeki K, Matsumoto S, Wakatsuki K, Enomoto K, Tanaka T, Ito M, Kurumatani N and Nakajima Y: The prognostic nutritional index predicts long-term outcomes of gastric cancer patients independent of tumor stage. Ann Surg Oncol 20(8): 2647-2654, 2013.

8 Schwegler I, von Holzen A, Gutzwiller JP, Schlumpf R, Muhlebach $\mathrm{S}$ and Stanga $\mathrm{Z}$ : Nutritional risk is a clinical predictor of postoperative mortality and morbidity in surgery for colorectal cancer. Br J Surg 97(1): 92-97, 2010.

9 Proctor MJ, Morrison DS, Talwar D, Balmer SM, Fletcher CD, O'Reilly DS, Foulis AK, Horgan PG and McMillan DC: A comparison of inflammation-based prognostic scores in patients with cancer. A glasgow inflammation outcome study. Eur J Cancer 47(17): 2633-2641, 2011.

10 Sun $\mathrm{K}$, Chen S, Xu J, Li G and He Y: The prognostic significance of the prognostic nutritional index in cancer: A systematic review and meta-analysis. J Cancer Res Clin Oncol 140(9): 1537-1549, 2014.

11 Association JGC: Japanese gastric cancer treatment guidelines 2010 (ver. 3). Gastric Cancer 14(2): 113-123, 2011.

12 Association JGC: Japanese classification of gastric carcinoma: 3rd english edition. Gastric Cancer 14(2): 101-112, 2011.

13 Dindo D, Demartines $\mathrm{N}$ and Clavien PA: Classification of surgical complications: A new proposal with evaluation in a cohort of 6336 patients and results of a survey. Ann Surg 240(2): 205-213, 2004.

14 Li QG, Li P, Tang D, Chen J and Wang DR: Impact of postoperative complications on long-term survival after radical resection for gastric cancer. World J Gastroenterol 19(25): 40604065, 2013.

15 Tokunaga M, Tanizawa Y, Bando E, Kawamura T and Terashima M: Poor survival rate in patients with postoperative intraabdominal infectious complications following curative gastrectomy for gastric cancer. Ann Surg Oncol 20(5): 1575-1583, 2013.

16 Kubota T, Hiki N, Sano T, Nomura S, Nunobe S, Kumagai K, Aikou S, Watanabe R, Kosuga T and Yamaguchi T: Prognostic significance of complications after curative surgery for gastric cancer. Ann Surg Oncol 21(3): 891-898, 2014. 
17 Hayashi T, Yoshikawa T, Aoyama T, Hasegawa S, Yamada T, Tsuchida K, Fujikawa H, Sato T, Ogata T, Cho H, Oshima T, Rino $\mathrm{Y}$ and Masuda M: Impact of infectious complications on gastric cancer recurrence. Gastric Cancer 18(2): 368-374, 2015.

18 Imamura H, Nishikawa K, Kishi K, Inoue K, Matsuyama J, Akamaru Y, Kimura Y, Tamura S, Kawabata R, Kawada J, Fujiwara Y, Kawase T, Fukui J, Takagi M, Takeno A and Shimokawa $\mathrm{T}$ : Effects of an oral elemental nutritional supplement on post-gastrectomy body weight loss in gastric cancer patients: A randomized controlled clinical trial. Ann Surg Oncol 23(9): 2928-2935, 2016.

19 Kubo H, Komatsu S, Ichikawa D, Kawaguchi T, Kosuga T, Okamoto K, Konishi H, Shiozaki A, Fujiwara H and Otsuji E: Impact of body weight loss on recurrence after curative gastrectomy for gastric cancer. Anticancer Res 36(2): 807-813, 2016.

20 Aoyama T, Yoshikawa T, Shirai J, Hayashi T, Yamada T, Tsuchida K, Hasegawa S, Cho H, Yukawa N, Oshima T, Rino Y, Masuda M and Tsuburaya A: Body weight loss after surgery is an independent risk factor for continuation of s-1 adjuvant chemotherapy for gastric cancer. Ann Surg Oncol 20(6): 2000-2006, 2013.
21 Sietses C, Beelen RH, Meijer S and Cuesta MA: Immunological consequences of laparoscopic surgery, speculations on the cause and clinical implications. Langenbecks Arch Surg 384(3): 250258, 1999.

22 Goldfarb Y, Sorski L, Benish M, Levi B, Melamed R and BenEliyahu S: Improving postoperative immune status and resistance to cancer metastasis: A combined perioperative approach of immunostimulation and prevention of excessive surgical stress responses. Ann Surg 253(4): 798-810, 2011.

Received March 9, 2018

Revised March 29, 2018

Accepted April 3, 2018 